\title{
Una década del mensaje sobre la salud en las revistas de comunicación indexadas en IN-RECS (2000-2011)
}

\author{
Roberto MARTÍNEZ-PECINO \\ Universidad de Sevilla \\ rmpecino@us.es \\ Ramón REIG \\ Universidad de Sevilla \\ ramonreig@us.es \\ Manuel MARÍN-SÁNCHEZ \\ Universidad de Sevilla \\ mmsanchez@us.es
}

Recibido: 07/02/2013

Aceptado: 09/09/2013

\begin{abstract}
Resumen
Este trabajo analiza el abordaje que realizan las revistas científicas de comunicación con mayor impacto en el índice IN-RECS del mensaje sobre la salud a lo largo de la última década. Se revisaron 1.962 referencias analizando frecuencia de publicación, autoría, temática, medios y tipo de análisis realizado. Se observó escasa presencia de estudios sobre el mensaje de la salud en las revistas de comunicación. La mayor parte trataron sobre salud pública centrándose bien en enfermedades concretas o en aspectos que pueden afectar a gran parte de la población. La mayoría de los estudios realizaron análisis de contenidos principalmente de prensa, seguidos de contenidos audiovisuales y con menor frecuencia Internet; en menor medida se narraron experiencias o reflexiones. Finalmente, realizamos propuestas de investigación para seguir contribuyendo al progreso de este ámbito.

Palabras clave: mensaje sobre salud, medios de comunicación, revistas de comunicación, periodismo médico.

\section{A decade of health message in communication journals indexed in IN-RECS (2000-2011)}

\begin{abstract}
The aim of this paper is to analyse how scientific communication journals with greater impact factor on the IN-RECS index approach health message over the last decade. A total of 1.962 references were reviewed by analyzing publication frequency, authorship, topics, media considered and type of analysis made. It was observed scarcity of studies about health message in communication journals. Most of the articles dealt with public health issues focusing either on specific diseases or on issues that can affect much of the population. Most studies conducted content analysis mainly of press followed by audiovisual content and Internet sparingly; to a lesser extent narrated experiences or reflections. Lines of research are also proposed to continue contributing to progress in this area.

Keywords: health message, mass media, communication journals, medical journalism.

\section{Referencia normalizada}

MARTÍNEZ-PECINO, Roberto; REIG, Ramón; y MARÍN-SÁNCHEZ, Manuel (2013): “Una década del mensaje sobre la salud en las revistas de comunicación indexadas en IN-RECS (2000-2011)". Estudios sobre el Mensaje Periodístico. Vol. 19, Núm. 2 (julio-diciembre), págs.: 1047-1059. Madrid, Servicio de Publicaciones de la Universidad Complutense.
\end{abstract}

Sumario: 1. Introducción. 2. Metodología; 2.1. Muestra y periodo de estudio; 2.2. Procedimiento. 3. Resultados. 4. Discusión y conclusiones. 5. Referencias. 


\section{Introducción}

Los medios de comunicación prestan cada vez más atención a los temas de la salud ya que la sociedad demanda de forma progresiva información sanitaria. De esta forma se favorece que haya más variedad de este tipo de información en los medios (Moreno, 2010) así como la incorporación de secciones específicas sobre salud en diarios, televisión, radio e Internet (Santos, 2009). Del mismo modo los medios ejercen una notable influencia sobre la sociedad (Sabucedo y Rodríguez, 1997) a la que el ámbito de la salud no es ajena (Ertem et al., 2010; Lu y Yang, 2007) y que se produce ya sea por su exposición directa o de modo indirecto a través de la conciencia social que generan en otros (Niederdeppe et al., 2007). La influencia se manifiesta al menos desde tres perspectivas: mediante el establecimiento de la agenda (agenda setting), mediante la teoría del cultivo y mediante el aprendizaje social.

Un aspecto básico de los medios es su capacidad para establecer temas de la agenda pública (McCombs y Shaw, 1972; Lopez-Escobar et al., 1998). De esta forma, la agenda nos ayuda, entre otros aspectos, a determinar sobre qué pensar. La teoría del cultivo (cultivation theory) establece que los medios nos ayudan a desarrollar cómo pensar sobre los temas (Gerbner et al., 2002). Finalmente la teoría del aprendizaje social nos indica que las personas aprendemos a comportarnos mediante la observación, directa o en los medios, del comportamiento de otros; en este sentido es más probable imitar el comportamiento de aquellos que nos resultan atractivos, son similares a nosotros, y sus comportamientos resultan recompensados (Bandura, 1977; Bandura, 2001).

Los profesionales del sector sanitario han tomado conciencia de la importancia de la relación entre la salud y la comunicación tanto en lo que se refiere a la comunicación asistencial médico-paciente como a la información que aparece en los medios de comunicación. Un ejemplo del interés por la información sobre salud aparecida en los medios se muestra en la creciente presencia de esta temática en revistas científicas médicas. Así, por ejemplo, ya en 1998 encontramos un editorial de la revista British Medical Journal (Smith, 1998) dedicado al análisis de la relación entre salud y los medios de comunicación. Más recientemente, en 2011, en un editorial de Gaceta Sanitaria se reflexionaba sobre las estrategias de marketing y comunicación de la industria farmacéutica para incrementar la demanda de sus productos (Ruiz-Cantero y CambroneroSaiz, 2011). En Archives of Internal Medicine, mediante un estudio en el que se encuestaba a 6.369 personas, se ponía de manifiesto que Internet se ha convertido en uno de los medios de referencia utilizados por la población a la hora de informarse sobre la salud así como el papel cada vez más importante de los medios de comunicación como fuente de información médica para la población (Hesse et al., 2005).

Las revistas científicas de comunicación son el foro de reflexión entre académicos y expertos de comunicación social de todo el mundo. Con frecuencia analizan la cobertura en los diferentes medios de temas que resultan de relevancia social como por ejemplo la política (Barreto et al., 2010), la inmigración (Muñiz et al., 2006) o la economía (Arrese, 2000). La salud es un tema de gran relevancia ya que conlleva implicaciones personales, sociales, económicas y políticas (Díaz, 2009).

Nos planteamos como pregunta de investigación si las revistas científicas de comunicación reflejan el interés profesional y social respecto al mensaje sobre la salud 
en los medios. Por ello, el objetivo de este trabajo es analizar el abordaje que realizan las revistas científicas con mayor impacto en la categoría de comunicación del mensaje sobre la salud a lo largo del periodo 2000-2011.

\section{Metodología}

\subsection{Muestra y periodo de estudio}

Se revisaron todos los artículos, reseñas y apuntes, publicados en las revistas científicas de la categoría de comunicación situadas en el primer cuartil del índice IN-RECS. Como periodo de análisis se consideró la última década, desde enero del 2000 hasta septiembre de 2011.

El índice IN-RECS es un indicador de la influencia, relevancia e impacto de las revistas de Ciencias Sociales en España elaborado por el Grupo de Evaluación de la Ciencia y de la Comunicación Científica de la Universidad de Granada. Se utilizó este indicador por tener gran reconocimiento a nivel nacional (Giménez y Alcain, 2006; Castillo y Carreton, 2010).

En la tabla 1, se muestra el listado de revistas en la categoría de comunicación situadas en el primer cuartil del índice IN-RECS, actualizado a 2009, que es el año más actual disponible en el momento en que se elaboró este trabajo y su índice de impacto.

Tabla 1. Revistas en la categoría de comunicación en el primer cuartil del índice IN-RECS actualizado a 2009 y su índice de impacto

\begin{tabular}{|c|c|l|r|}
\hline Cuartil & Posición & Título de la revista & Indice impacto 2009 \\
\hline $1^{\circ}$ & 1 & Revista Latina de Comunicación Social & 1.380 \\
\hline $1^{\circ}$ & 2 & Comunicación y sociedad & 0.428 \\
\hline $1^{\circ}$ & 3 & Zer. Revista de Estudios de Comunicación & 0.357 \\
\hline $1^{\circ}$ & 4 & Estudios sobre el mensaje periodístico & 0.252 \\
\hline $1^{\circ}$ & 5 & CIC. Cuadernos de información y comunicación & 0.250 \\
\hline
\end{tabular}

\subsection{Procedimiento}

Se accedió a las revistas a través de los sitios web de las mismas y de la base Dialnet. Dos investigadores de manera independiente revisaron la información disponible acerca del título, resumen y palabras claves de las cinco revistas mencionadas desde enero del 2000 a septiembre de 2011. Los revisores fueron un psicólogo que realiza actividades docentes e investigadoras en la Facultad de Ciencias de la Comunicación y una médica con formación y experiencia en investigación y que trabaja en el área de la salud pública. Se aportó de esta forma una visión multidisciplinar.

Se realizó una primera selección amplia con la intención de identificar todos los documentos relacionados con la salud. Una vez seleccionados fueron analizados a texto completo. Los que presentaban alguna duda eran discutidos por los revisores hasta llegar a un acuerdo. No se precisó la intervención de un tercer revisor, ya que tras la discusión siempre se alcanzó un acuerdo.

Se consideró como definición de salud la aportada por la Organización Mundial de la Salud que la define del siguiente modo:

La salud es un estado de completo bienestar físico, mental y social, y no solamente la ausencia de afecciones o enfermedades". La cita procede del Preámbulo de la Constitución de la Organización Mundial de la Salud, que fue adoptada por la Conferencia Sanita- 
ria Internacional, celebrada en Nueva York del 19 de junio al 22 de julio de 1946, firmada el 22 de julio de 1946 por los representantes de 61 Estados (Official Records of the World Health Organization, $\mathrm{N}^{\mathrm{o}} 2$, p. 100), y entró en vigor el 7 de abril de 1948. La definición no ha sido modificada desde 1948 (cita textual extraída del siguiente enlace http://www.who.int/suggestions/faq/es/).

En consonancia con la amplia definición de salud se hizo operativo el trabajo considerando los siguientes criterios de inclusión: cualquier artículo independientemente del diseño, incluyendo reseñas, apuntes u otro tipo de publicaciones recogidas en las revistas objeto de estudio que incluyesen en el título, resumen o palabras claves, términos relacionados con salud, enfermedad, síntomas, atención sanitaria, estilos de vida saludable, salud pública, industria farmacéutica, calidad de vida, gestión sanitaria, investigación biomédica, tecnologías sanitarias, profesionales o usuarios de servicios sanitarios.

Se excluyeron aquellos documentos en los que, aunque utilizaran términos relacionados con la salud, el contenido del artículo no estuviera relacionado con este tema o bien aunque el estudio pudiera tener implicaciones para la salud no fuera ésta el objetivo del mismo.

Una vez seleccionados los documentos que se incluyeron en el estudio se analizó la frecuencia de aparición, autoría, temática de los documentos, la relación con algún medio de comunicación y la aproximación o análisis realizado.

\section{Resultados}

Se revisaron en total 1.962 referencias. Se seleccionaron inicialmente a texto completo 29 documentos. Tras la lectura se excluyeron 12, identificándose finalmente 17 documentos relacionados con la salud. Los artículos relacionados con salud supusieron el $0,86 \%$ de los documentos publicados en las cinco revistas incluidas en el primer cuartil de la categoría de comunicación en el índice IN-RECS actualizado a 2009.

De los artículos incluidos, el 29,41 \% pertenecen a Revista Latina de Comunicación Social, el 11,76\% a Comunicación y Sociedad, el 23,52 \% a Zer. Revista de Estudios de Comunicación, y el 35,29\% a Estudios sobre el Mensaje Periodístico, siendo por tanto esta revista la que más atención dedica a esta temática. En la revista CIC. Cuadernos de información y Comunicación no se encontró ningún artículo sobre la salud.

El 68,18\% de los autores de los documentos que finalmente se incluyeron en el estudio eran mujeres.

En la tabla 2 se muestra el número de artículos sobre salud publicados cada año en las revistas objeto de estudio. Los documentos que se publicaron en el 2009 suponen el $35,29 \%$ de los publicados. Llama la atención que en algunos años como en 2008, 2006 y 2003 no se publicó ningún documento relacionado con la salud. En la tabla 3 se recogen para cada una de las revistas los títulos de los

\begin{tabular}{|c|c|c|c|}
\hline \multicolumn{3}{|c|}{ Tabla 2. Número de artículos sobre } \\
salud publicados cada año.
\end{tabular}


documentos que se incluyeron finalmente en el estudio, indicando el año de publicación de los mismos.

\begin{tabular}{|c|c|c|}
\hline Revistas & Año & Título \\
\hline \multirow{5}{*}{$\begin{array}{l}\text { Revista Latina de } \\
\text { Comunicación Social }\end{array}$} & 2009 & "La 'gripe A', en la prensa española" \\
\hline & 2009 & $\begin{array}{l}\text { "Teleasistencia no invasiva mediante televisión } \\
\text { digital: estudio de los hábitos de consumo de } \\
\text { televisión de los mayores" }\end{array}$ \\
\hline & 2009 & $\begin{array}{l}\text { "Cómo cambia el marketing la imagen social de } \\
\text { la cocaína" }\end{array}$ \\
\hline & 2002 & $\begin{array}{l}\text { "Gestión del conocimiento en el primer nivel de } \\
\text { atención de salud, en Heredia (Costa Rica)" }\end{array}$ \\
\hline & 2001 & "Drogas y comunicación" \\
\hline \multirow[t]{2}{*}{ Comunicación y sociedad } & 2007 & $\begin{array}{l}\text { "Propuesta de un modelo para el seguimiento de } \\
\text { un tema en la prensa: análisis de textos } \\
\text { periodísticos sobre el cuidado de las personas en } \\
\text { su entorno familiar" }\end{array}$ \\
\hline & 2000 & $\begin{array}{l}\text { "La función del recuerdo de los medios. La } \\
\text { cobertura de grandes temas" }\end{array}$ \\
\hline \multirow{4}{*}{$\begin{array}{l}\text { Zer. Revista de Estudios de } \\
\text { Comunicación }\end{array}$} & 2009 & $\begin{array}{l}\text { “Marcas o genéricos? La comunicación en línea } \\
\text { de las marcas farmacéuticas” }\end{array}$ \\
\hline & 2002 & $\begin{array}{l}\text { "La prevención del SIDA mediante cortos de } \\
\text { ficción. Una investigación experimental" }\end{array}$ \\
\hline & 2000 & $\begin{array}{l}\text { "Influencias mediáticas y personales sobre la } \\
\text { decisión de protegerse del VIH/SIDA" }\end{array}$ \\
\hline & 2000 & $\begin{array}{l}\text { "De la detección al recuerdo: Una historia } \\
\text { periodística del SIDA" }\end{array}$ \\
\hline \multirow{6}{*}{$\begin{array}{l}\text { Estudios sobre el mensaje } \\
\text { periodistico }\end{array}$} & 2011 & "La obesidad infantil en la prensa Española"l \\
\hline & 2010 & $\begin{array}{l}\text { "Periodismo biomédico, nuevos contenidos } \\
\text { mediáticos" }\end{array}$ \\
\hline & 2009 & $\begin{array}{l}\text { "El conceptismo como recurso retórico en } \\
\text { columnas periodísticas personales. Análisis de } \\
\text { un tema: la obesidad" }\end{array}$ \\
\hline & 2009 & $\begin{array}{l}\text { "La salud en la prensa gratuita especializada. Un } \\
\text { fenómeno emergente" }\end{array}$ \\
\hline & 2005 & $\begin{array}{l}\text { "Enfermedad del Papa y credibilidad } \\
\text { periodística. Atribución de fuentes durante los } \\
\text { últimos días de Juan Pablo II" }\end{array}$ \\
\hline & 2004 & $\begin{array}{l}\text { "La crisis de las vacas locas en España: la } \\
\text { necesidad de una información sanitaria } \\
\text { responsable" }\end{array}$ \\
\hline
\end{tabular}

${ }^{1}$ En el momento del estudio de este artículo estaba disponible el título y la autoría pero no el texto completo por lo que en la sección de resultados no se tuvo en cuenta para valorar la parte correspondiente a la relación con algún medio de comunicación y la aproximación o análisis realizado. 
CIC. Cuadernos de información y comunicación
Ninguno

En cuanto a la temática de estos documentos pueden ser agrupados en tres grandes secciones. En primer lugar, los que versan sobre aspectos de salud pública centrándose ya sea en enfermedades concretas o en aspectos de salud que pueden afectar a gran parte de la población. Estos artículos suponen la mayor parte (76,47\%); entre ellos los temas más tratados son los relacionados con el sida, la drogadicción y la obesidad. En segundo lugar, encontramos aquellos artículos en los que la salud se trata de manera genérica $(17,64 \%)$ sin centrarse en enfermedades ni contenidos concretos, y finalmente un artículo $(5,88 \%)$ que trata sobre la industria farmacéutica.

La mayor parte de los documentos se centran en análisis de prensa $(58,82 \%)$ ya sea impresa o digital, le sigue el análisis de contenidos audiovisuales $(23,52 \%)$ y de Internet $(5,88 \%)$. Los trabajos realizan principalmente $(70,58 \%)$ análisis de contenidos, considerando cómo se enmarcan los temas de salud en los medios; en menor medida se narran experiencias o reflexiones.

En la tabla 4 se muestra el listado con los títulos de los documentos excluidos tras el análisis del contenido del texto completo. Se muestra también el año y revista en la que se publicaron.

\begin{tabular}{|c|c|c|}
\hline Revistas & Año & Título \\
\hline \multirow{5}{*}{$\begin{array}{l}\text { Revista Latina de } \\
\text { Comunicación } \\
\text { Social }\end{array}$} & 2009 & $\begin{array}{l}\text { "Series familiares de televisión: concepto, producción y } \\
\text { exportación. El caso de Médico de familia" }\end{array}$ \\
\hline & 2009 & $\begin{array}{l}\text { "Pedagogías del cuerpo. La construcción espec(tac)ular del } \\
\text { cuerpo femenino en el reality show español" }\end{array}$ \\
\hline & 2008 & $\begin{array}{l}\text { "Características de la figura humana en los spots de bebidas } \\
\text { emitidos en España en } 2006 "\end{array}$ \\
\hline & 2003 & $\begin{array}{l}\text { "El hombre, en el anuncio publicitario. Un ejemplo práctico: el } \\
\text { anciano" }\end{array}$ \\
\hline & 2000 & "Internet: un cuerpo enfermo y un campo de batalla" \\
\hline $\begin{array}{l}\text { Comunicación y } \\
\text { sociedad }\end{array}$ & 2008 & $\begin{array}{l}\text { "Series de televisión. El caso de Médico de familia, Cuéntame } \\
\text { cómo pasó y Los Serrano" }\end{array}$ \\
\hline \multirow{4}{*}{$\begin{array}{l}\text { Zer. Revista de } \\
\text { Estudios de } \\
\text { Comunicación }\end{array}$} & 2011 & $\begin{array}{l}\text { "Application of Biofeedback in the Training of Radio and } \\
\text { Television Broadcasters" }\end{array}$ \\
\hline & 2011 & $\begin{array}{l}\text { "La metáfora visual corporeizada. Bases cognitivas del discurso } \\
\text { audiovisual" }\end{array}$ \\
\hline & 2009 & $\begin{array}{l}\text { "Comunicación pública de la tecnociencia: más allá de la } \\
\text { difusión del conocimiento" }\end{array}$ \\
\hline & 2004 & $\begin{array}{l}\text { "Medicina Preventiva en tiempos de crisis, basada en la gestión } \\
\text { de la marca-empresa" }\end{array}$ \\
\hline $\begin{array}{l}\text { Estudios sobre el } \\
\text { mensaje periodístico }\end{array}$ & 2009 & $\begin{array}{l}\text { "La rehabilitación neurocientífica de la empatía y sus } \\
\text { implicaciones en los ámbitos de la comunicación" }\end{array}$ \\
\hline $\begin{array}{l}\text { CIC. Cuadernos de } \\
\text { información y } \\
\text { comunicación }\end{array}$ & 2009 & "La homeostasis familiar y el médico" \\
\hline
\end{tabular}


Aunque los títulos de los artículos excluidos podrían hacer pensar que se refieren a la salud, se descartaron tras su lectura detallada. En ocasiones los términos se utilizaron de forma metafórica, por ejemplo, en la revista Zer. Revista de Estudios de Comunicación, el artículo titulado "Medicina preventiva en tiempos de crisis, basada en la gestión de la marca-empresa". Si bien la palabra medicina se utilizó en el título su contenido la empleaba tan sólo metafóricamente, reflexionando el documento acerca de la gestión de la marca de la empresa como elemento corporativo que puede actuar como medicina empresarial en tiempos de crisis. Otro de los documentos publicados en esta revista titulado "Comunicación pública de la tecnociencia: más allá de la difusión del conocimiento", aunque en algún momento hacía algunas breves menciones a la salud, su contenido en términos generales no era un análisis de dichos temas, sino más bien una reflexión acerca de la información científica (no sólo de salud) y tecnológica en los medios. En otro de los documentos, publicado en Comunicación y Sociedad, la reseña referente a las "Series de televisión. El caso de Médico de familia, Cuéntame cómo pasó y Los Serrano" aunque en el título refería literalmente la expresión médico de familia, su contenido no se articulaba a un análisis de la profesión médica en la serie, sino más bien a valorar qué factores ayudan a comprender el éxito de estas tres series.

\section{Discusión y conclusiones}

A pesar de la relevancia de los temas relacionados con la salud en todos los estamentos sociales, una de las conclusiones derivadas del estudio y análisis de datos realizados es que se observa una escasa presencia de trabajos que aborden el mensaje sobre la salud en las revistas de comunicación situadas en el primer cuartil del índice INRECS en la década estudiada, suponiendo menos de un $1 \%$ de los documentos publicados. La revista Estudios Sobre el Mensaje Periodístico es la que tiene un mayor porcentaje de artículos sobre el mensaje de la salud, seguida por la Revista Latina de Comunicación Social, la revista Zer. Revista de Estudios de Comunicación y la revista Comunicación y Sociedad. En la revista CIC. Cuadernos de Información y Comunicación no se identificó ningún documento sobre salud en la década analizada.

La mayor parte de los artículos publicados tratan sobre salud pública, centrándose bien en enfermedades concretas o en aspectos que pueden afectar a gran parte de la población, destacando como temas el SIDA, la drogadicción y la obesidad. Como comentábamos en la introducción, las revistas de comunicación con frecuencia abordan cómo son tratados en diferentes medios temas relevantes. Tanto el SIDA, como la drogadicción o la obesidad pueden considerarse temas relevantes por sus implicaciones sociales, personales, económicas y políticas. Además, se encuentran entre los diez temas en los que se centran más de la mitad de todo lo publicado sobre temas de salud en el ABC, El Mundo, El País, La Vanguardia y el Periódico de Catalunya desde 1997 al 2006 (Revuelta y De Semir, 2008). Otro de los artículos identificados trataba sobre la gripe $\mathrm{A}$, que también ha sido un tema con gran impacto mediático llegando a ser considerada como la gran noticia sanitaria de la primera década del siglo XXI (Fundación Vila Casas, 2011). 
Que los temas más recogidos en las revistas de comunicación coincidan con las cuestiones más mediáticas es significativo ya que marcan la agenda social. La relevancia de los medios en relación a la agenda setting se ha mostrado también en otros ámbitos de investigación (López-Escobar y Llamas, 1996; López-Escobar et al., 1996). Sin embargo, es conveniente tener presente que el hecho de que los temas de salud se concentren en unos pocos, no tiene por qué coincidir con los temas que preocupan a los profesionales sanitarios, o que implique que estos magnifiquen el tema de la misma forma. Por tanto, también se podría explorar en futuras investigaciones qué temas preocupan a los profesionales sanitarios y no son abordados en los medios de comunicación. Sería de utilidad también reflejar los efectos de distintos tipos de encuadre, por ejemplo, si es más eficaz para influir en las personas facilitar información de corte cognitivo o emocional (Dunlop et al., 2010, Guadarrama y Valero, 2006). Igualmente, valorar la presencia y efectos de diversos tipos de información como aquella que resulta alarmista frente a otros enfoques basados en desarrollar estrategias de afrontamiento (Chang, 2011).

La mayor parte de los artículos relacionados con salud se publicaron en las revistas científicas de comunicación en 2009, 2002 y 2000. Sorprende que en algunos años (2008, 2006 y 2003) no se haya publicado ningún artículo relacionado con la salud. Una posible explicación podría ser que hubiese grupos de investigación en salud que publicasen cada cierto tiempo. Esto lo descartamos tras revisar los autores de los artículos y comprobar que no se repetían.

Los documentos que se incluyeron en el estudio realizaron análisis de contenidos sobre todo de prensa, seguidos de contenidos audiovisuales y muy escasamente de Internet. Resulta relevante que los estudios, aunque sean escasos, recogen los principales medios de comunicación. No obstante, sería interesante profundizar en mayor medida sobre la cobertura de la salud en Internet (Rains, 2008) ya que está jugando un papel cada vez más importante como fuente de información médica (Hesse, et al., 2005) para la población de diferentes edades (Martinez-Pecino et al., 2011; Zhao, 2009) siendo la calidad de la misma muy variada (Moraham-Martin, 2004; Sillence et al., 2007). Otro aspecto a tener en cuenta en futuras investigaciones es el incremento de contenidos generados por los usuarios en la red (User Generated Contents) (Nieto, 2008; Yoo y Kim, 2011). En este sentido puede ser útil comparar la credibilidad y efectos de diversas fuentes de las que proviene la información tales como fuentes institucionales, profesionales o personales (Hu y Sundar, 2010).

El objetivo de este estudio era explorar el abordaje que realizan las revistas de comunicación con mayor impacto en el índice IN-RECS acerca del mensaje sobre la salud en el periodo 2000-2011. La revisión realizada y los resultados derivados de la misma nos han permitido conocer la frecuencia de estas publicaciones, distribución en años de las mismas, autoría, las temáticas de los documentos, los medios de comunicación que han considerado y la aproximación o tipo de análisis que han realizado. Con ello hemos contribuido a desarrollar un mayor conocimiento acerca del abordaje del mensaje sobre la salud en las revistas científicas de comunicación y se han sugerido también algunas líneas de investigación con las que continuar la investigación en este ámbito. 


\section{Referencias}

ALCÍBAR, Miguel (2009): “Comunicación pública de la tecnociencia: más allá de la difusión del conocimiento." Zer, Revista de Estudios de Comunicación, 14(27), pp. 165-188.

ARRESE, Ángel (2000): "Economía y medios de comunicación en la década de los setenta". Comunicación y Sociedad, 13(2), pp. 9-51.

BANDURA, Albert (1977): Social learning theory. Englewood, Cliffs:NJ, PrenticeHall.

BANDURA, Albert (2001): "Social Cognitive Theory of Mass Communication". Media Psychology, 3(3), pp. 265-299.

BARRETO, Idaly; OROZCO, Henry Borja; SABUCEDO, José Manuel; LÓPEZLÓPEZ, Wilson; y ALZATE Mónica (2010): "Creencias legitimadoras y deslegitimadotas difundidas por la prensa española sobre el asesinato político". Revista Latinoamericana de Psicología, 42(3), pp. 437-452.

CAMACHO, Idoia (2009): “La 'gripe A', en la prensa española” Revista Latina de Comunicación Social, no 64, pp.827-843.

CASTILLO, Antonio y CARRETÓN, María Carmen (2010): "Investigación en Comunicación. Estudio bibliométrico de las Revistas de Comunicación en España". Comunicación y Sociedad, 23(2), pp. 289-327.

CARIILLO, María Victoria (2004): "Medicina Preventiva en tiempos de crisis, basada en la gestión de la "marca-empresa". Zer. Revista de Estudios de Comunicación, $\mathrm{n}^{\circ} 17$, pp. 67-83.

CHANG, Chingching (2011): "News coverage of health-related issues and its impacts on perceptions. Taiwan as an example." Health Communication, 27(2), pp. 111-123.

COLLE, Raymond (2000): "Internet: un cuerpo enfermo y un campo de batalla". Revista Latina de Comunicación Social, 30, disponible en: http://www.ull.es/publicaciones/latina/aa2000qjn/91 colle.htm.

DÍAZ, José Antonio (2009): “El conceptismo como recurso retórico en columnas periodísticas personales. Análisis de un tema: la obesidad". Estudios sobre el Mensaje Periodístico, $\mathrm{n}^{\circ}$ 15. Madrid, Servicio de Publicaciones de la Editorial Complutense, pp. 207-226.

DUNLOP, Sally; WAKEFIELD, Melanie; \& KASHIMA, Yoshihisa (2010): "Pathways to persuasion. Cognitive and experiential responses to health-promoting mass media messages". Communication Research, 37(1), pp. 133-164.

ERTEM, Gul; CANDAN, Yelda; \& OKSEL, Esra (2010): “An investigation of nursing news in Turkish daily newspaper”. Social Behavior and Personality, 38(5), pp. 577-582.

FUNDACIÓN VILA CASAS (2011): “Comunicación sanitaria. Gripe A”. Opinión Quiral, 31.1, 1-4. Publica Fundació Vila Casas, Observatori de la Comunicació Científica. 
GERBNER, George; GROSS, Larry; MORGAN, Michael; SIGNORIELLI, Nartty; \& SHANAHAN, James (2002): "Growing Up With Television. Cultivation Processes", en BRYANT, Jennings y ZILLMAN, Dolf: Media Effects. Advances in Theory and Research. Hillsdale, NJ, Erlbaum, pp. 43-67.

GIMÉNEZ, Elea y ALCAÍN, María Dolores (2006): "Estudio de las revistas españolas de periodismo". Comunicación y Sociedad, 19(2), pp.107-131.

GUADARRAMA, Luis Alberto y VALERO, Janet (2006): "Estrategias edu-comunicacionales para mejorar la salud adolescente". Comunicar, n' 26, pp.51-57.

HERNÁNDEZ, Ana (2001): "Drogas y comunicación”. Revista Latina de Comunicación Social, $n^{\circ} 45$, pp. 1-4.

HERRERO, Mónica y DIEGO, Patricia (2009): "Series familiares de televisión: concepto, producción y exportación. El caso de Médico de familia". Revista Latina de Comunicación Social, nº 64, pp. 238-247.

HESSE, Bradford.W; NELSON, David; KREPS, Gary; CROYLE, Robert; ARORA, Neeraj; RIMER, Barbara; \& VISWANATH, Kasisomayajula (2005): "Trust and Sources of Health Information: The Impact of the Internet and Its Implications for Health Care Providers: Findings From the First Health Information National Trends Survey". Archives of Internal Medicine, 165(22), pp. 2618-2624.

HU, Yifeng \& SUNDAR, Shyam (2010): "Effects of online health sources on credibility and behavioral intentions". Communication Research, 37(1), pp.105-132.

HUERTAS, Assumpció y XIFRA, Jordi (2009): “¿Marcas o genéricos? La comunicación en línea de las marcas farmacéuticas". Zer, Revista de Estudios de Comunicación, 14(27), pp. 251-270.

IGARTUA, Juan José y LOPES, Orquídea (2002): "La prevención del SIDA mediante cortos de ficción. Una investigación experimental". Zer, Revista de Estudios de Comunicación, 13, disponible en http://www.ehu.es/zer/zer13/sida13.htm.

JACKSON, Deavila (2009): "La homeostasis familiar y el médico". CIC. Cuadernos de información y comunicación, 14, pp.15-20.

KORNBLIT, Ana Lia y PETRACCI, Mónica (2000): "Influencias mediáticas y personales sobre la decisión de protegerse del VIH/SIDA". Zer, Revista de Estudios de Comunicación, 8, disponible en http://www.ehu.es/zer/zer8/8lia2.html.

LEÓN, Reyes (2003): "El hombre, en el anuncio publicitario. Un ejemplo práctico: el anciano". Revista Latina de Comunicación Social, 56, disponible en: http://www.ull.es/publicaciones/latina/20035631leon.htm

LÓPEZ-ESCOBAR, Esteban y LLAMAS, Juan Pablo (1996): "Agenda-setting: investigaciones sobre el primero y el segundo nivel". Comunicación y Sociedad, $9(1$ y 2), pp. 9-15.

LÓPEZ-ESCOBAR, Esteban; LLAMAS, Juan Pablo; \& MCCOMBS, Maxwell (1998): "Agenda-setting and community consensus: first and second level effects". International Journal of Public Opinion Research, 10(4), pp. 335-348. 
LÓPEZ-ESCOBAR, Esteban; LLAMAS, Juan Pablo; y REY, Federico (1996): "La agenda entre los medios: primero y segundo nivel". Comunicación y Sociedad, 9(1 у 2), pp. 67-89.

LU, Jane. \& YANG, Shuen-An (2007): "Who is watching Tv? Who is listening to radio? Consumer perceptions of Tv and radio advertising information" Social Behavior and personality, 35(2), pp.157-167.

MARTÍN, Marta (2000): "De la detección al recuerdo: una historia periodística del SIDA. Zer, Revista de Estudios de Comunicación", 8, disponible en: http://www.ehu.es/zer/zer8/8martin3.html

MARTÍN, Marta (2000): "La función de recuerdo de los medios. Las coberturas de "grandes temas". Comunicación y Sociedad, 13(1), pp.115-138.

MARTÍNEZ SOLANA, Yolanda (2004): "La crisis de las vacas locas en España: la necesidad de una información sanitaria responsable". Estudios sobre el Mensaje Periodístico, $\mathrm{n}^{\circ}$ 10. Madrid, Servicio de Publicaciones de la Editorial Complutense, pp.139-158.

MARTÍNEZ-PECINO, Roberto; CABECINHAS, Rosa; \& LOSCERTALES-ABRIL, Felicidad (2011): "University senior students on the web". Comunicar, 37, pp. 8995.

MCCOMBS, Maxwell \& SHAW, Donald (1972): "The agenda setting function of mass media". Public Opinion Quarterly, 36(2), 176-187.

MORAHAN-MARTIN, Janet (2004): “ How Internet users find, evaluate, and use online health information. A cross-cultural review". Cyberpsychology \& Behavior, 7(5), 497-510.

MORENO, Pastora (2010): "Periodismo biomédico, nuevos contenidos mediáticos". Estudios sobre el Mensaje Periodístico. Vol. 16. Madrid, Servicio de Publicaciones de la Editorial Complutense, pp. 319-330.

MUÑIZ, Carlos; IGARTUA, Juan José; y Otero, José Antonio (2006): "Imágenes de la inmigración a través de la fotografía de prensa. Un análisis de contenido". Comunicación y Sociedad, 14(1), pp. 103-128.

NIEDERDEPPE, Jeff; Farrelly, Matthew; THOMAS, Kristin; WENTER, Dana; \& WEITZENKAMP, David (2007): "Newspaper coverage as indirect effects of a health communications intervention. The Florida tobacco control program and youth smoking". Communication Research, 34(4), pp. 382-406.

NIETO, Alfonso (2008): "Ciudadano y Mercado de la Comunicación". Comunicación y Sociedad, 21(2), pp. 7-33.

OJER, Teresa (2009): "Reseña de Series de televisión. El caso de médico de familia, Cuéntame como pasó y Los Serrano". Comunicación y Sociedad, 22(2).

ORTIZ, María (2011): "La metáfora visual corporeizada. Bases cognitivas del discurso audiovisual". Zer. Revista de Estudios de Comunicación, 16(30), pp. 57-73. 
PÉREZ-UGENA, Álvaro, SALAS, Álvaro; y VIZCAÍNO-LAORGA, Ricardo (2009): "Teleasistencia no invasiva mediante televisión digital. Estudio de los hábitos de consumo de televisión de los mayores". Revista Latina de Comunicación Social, $n^{0} 64$, pp. 632-645.

POU, María José (2005): "Enfermedad del Papa y credibilidad periodística. Atribución de fuentes durante los últimos días de Juan Pablo II". Estudios sobre el Mensaje Periodístico, $\mathrm{n}^{\mathrm{o}}$ 11. Madrid, Servicio de Publicaciones de la Editorial Complutense, pp. 113-128.

RAINS, Stephen (2008): "Health at high speed. Broadband internet access, health communication, and the digital divide", Communication Research, 35(3), pp. 283-297.

RAUSELL, Claudia (2008): "Características de la figura humana en los spots de bebidas emitidos en España en 2006". Revista Latina de Comunicación Social, n 63, pp. 220-237.

REVUELTA, Gema y DE SEMIR, Vladimir (2008): Medicina y Salud en la prensa diaria. Informe Quiral 10 años. Barcelona, Noclay.

RUIZ-CANTERO, María Teresa y CAMBRONERO-SAIZ, Belén (2011): "La metamorfosis de la salud: invención de enfermedades y estrategias de comunicación". Gaceta Sanitaria, n 25, 179-181.

SABUCEDO, José Manuel y RODRÍGUEZ, Miguel (1997): Medios de comunicación de masas y conducta política. Madrid, Biblioteca Nueva.

SÁNCHEZ, José Ramón (2009): "La rehabilitación neurocientífica de la empatía y sus implicaciones en los ámbitos de la comunicación”. Estudios sobre el mensaje periodístico, $\mathrm{n}^{\mathrm{o}}$ 15. Madrid, Servicio de Publicaciones de la Editorial Complutense, pp. 455-476.

SANTOS, María Teresa (2009): "La salud en la prensa gratuita especializada. Un fenómeno emergente". Estudios sobre el Mensaje Periodístico, $\mathrm{n}^{\circ} 15$. Madrid, Servicio de Publicaciones de la Editorial Complutense, pp. 477-490.

SAHUQUILLO, María (2007): "Propuesta de un modelo para el seguimiento de un tema en la prensa: análisis de textos periodísticos sobre el cuidado de las personas en su entorno familiar". Comunicación y Sociedad, 20(1), pp.103-144.

SILLENCE, Elisabeth; BRIGGS, Pamela; RICHARD Peter; \& FISHWICK, Leslye (2007): "How do patients evaluate and make use of online health information?". Social Science \& Medicine, 64(9), pp.1853-1862.

SIXTO, José y SALGUEIRO, María (2009): “Cómo cambia el marketing la imagen social de la cocaína". Revista Latina de Comunicación Social, nº 64, pp. 65-70.

SMITH, Richard (1998): "Editor's choice. Medical journals and the media". British Medical Journal, 316(7135), pp.1.

TORAL, Gotzon; GARCÍA, Irene; y MURELAGA, Jon (2011): "Application of Biofeedback in the Training of Radio and Television Broadcasters". Zer. Revista de Estudios de Comunicación, 16(30), pp. 115-133. 
VILLANUEVA, Luisa Elena (2002): "Gestión del conocimiento en el primer nivel de atención de salud, en Heredia (Costa Rica)". Revista Latina de Comunicación Social, $\mathrm{n}^{\circ} 52$, pp. 1-6.

WALZER, Alejandra (2009): "Pedagogías del cuerpo. La construcción espec(tac)ular del cuerpo femenino en el reality show español". Revista Latina de Comunicación Social, ${ }^{\circ}$ 64, pp. 203-209.

WESTALL, Debra (2011): "La obesidad infantil en la prensa española". Estudios sobre el mensaje periodístico, Vol. 17, núm. 1 (enero-junio). Madrid, Servicio de Publicaciones de la Universidad Complutense, pp. 225-239: http://dx.doi.org/10.5209/rev_ESMP.2011.v17.n1.13.

YOO, James \& KIM, Jinsang (2012): "Obesity in the new media. A content analysis of obesity videos on youtube". Health Communication, 27, pp. 86.97.

ZHAO, Shanyang (2009): "Parental education and children's online health information seeking: Beyond the digital divide debate". Social Science \& Medicine, 69(10), pp.1501-1505. 